\title{
-
}

Genctious

Journal of Neurogenetics

\section{Studying neural circuits of decision-making in Drosophila larva}

\section{Tihana Jovanic}

To cite this article: Tihana Jovanic (2020) Studying neural circuits of decision-making in Drosophila larva, Journal of Neurogenetics, 34:1, 162-170, DOI: 10.1080/01677063.2020.1719407

To link to this article: https://doi.org/10.1080/01677063.2020.1719407

册 Published online: 13 Feb 2020.

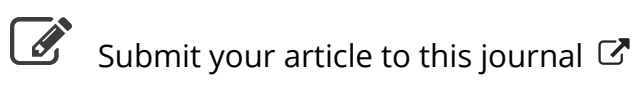

Шll Article views: 178

Q View related articles $\llbracket$

View Crossmark data $₫$ 


\title{
Studying neural circuits of decision-making in Drosophila larva
}

\author{
Tihana Jovanic ${ }^{\mathrm{a}, \mathrm{b}}$ \\ a Université Paris-Saclay, CNRS, Institut des Neurosciences Paris Saclay, Gif-sur-Yvette, France; ${ }^{\mathrm{b}}$ Decision and Bayesian Computation, UMR \\ 3571 Neuroscience Department \& USR 3756 (C3BI/DBC), Institut Pasteur \& CNRS, Paris, France
}

ABSTRACT

To study neural circuits underlying decisions, the model organism used for that purpose has to be simple enough to be able to dissect the circuitry neuron by neuron across the nervous system and in the same time complex enough to be able to perform different types of decisions. Here, I lay out the case: (1) that Drosophila larva is an advantageous model system that balances well these two requirements and (2) the insights gained from this model, assuming that circuit principles may be shared across species, can be used to advance our knowledge of neural circuit implementation of decision-making in general, including in more complex brains.
ARTICLE HISTORY

Received 22 August 2019

Accepted 18 January 2020

\section{KEYWORDS}

Drosophila larva; neural circuits; decision-making

\section{Introduction}

Any organism has the capacity to do many different behaviors, that all serve a certain purpose; from finding food, mating to escaping predators or avoiding other dangers. Yet many of these behaviors cannot occur simultaneously as they are physically mutually exclusive. An organism therefore needs to select which behavior to express in a given moment. The capacity to select the best behavior to execute is essential as depending on the situation some behaviors are more advantageous than others. If this process goes awry it can have serious consequences: if we don't eat when we are hungry or stay in presence of dangers we may die. Decisionmaking is therefore a fundamental aspect of behavior that is shared across the animal kingdom and the underlying mechanisms have evolved to ensure that it serves the organisms best interest and often combine instinctual drives and higher-order adaptive functions (Adams, Watson, Pearson, \& Platt, 2012; Kristan, 2008; Pearson, Watson, \& Platt, 2014).

The decision-making process needs to be successful in two ways. First, in terms of the appropriateness of the selected behavior to the situation. To make a choice, an animal will therefore take into account both the environmental context as wells as its internal physiological state (Palmer \& Kristan, 2011). It means the nervous system needs to be able to integrate different types of contextual and/or internalstate information with the predicted outcome of a behavior. This supposes an organization of the neural architecture that is flexible and where these systems can interact. Second, decisions need to be successfully implemented: the selected behavior needs to be fully expressed, while the competing behaviors need to be fully suppressed. Mechanisms must therefore exist in the nervous system that ensure that one behavior is promoted others are being inhibited (Kovac \&
Davis, 1977; Kupfermann \& Weiss, 2001; Redgrave, Prescott, \& Gurney, 1999). It is essential that this selection is full and complete so that the animals express clear and stable behaviors. Indeed, the etymology of the word decision stems from the Latin decider literally to cut off (for de-+ caedere 'to cut').

Ultimately, the problem of selecting a behavior would be very different if the nervous system were organized into discrete modules where specific sensory inputs generate behaviors executed by specific effectors (Kupfermann \& Weiss, 2001). Given that behaviors use overlapping neural networks and effector systems, the problem of resolving conflicts between competing demands on these systems by competing drives is a key feature of behavior (Prescott, 2007; Redgrave et al., 1999). Nervous systems therefore need to have mechanisms in place that can implement these competitive interactions successfully (Koyama \& Pujala, 2018; Maass, 2000; Mink, 1996; Redgrave, Vautrelle, \& Reynolds, 2011; Wang, 2008).

Studying cellular and synaptic mechanism of how the nervous system selects behaviors can be challenging in complex brains. Model systems with compact and numerically simple brains are more amenable for detailed characterization of the neural circuit mechanisms involved in competitive interactions (Baca, Marin-Burgin, Wagenaar, \& Kristan, 2008; Gaudry \& Kristan, 2009; Gillette \& Brown, 2015; Jovanic et al., 2016; Kovac \& Davis, 1977; Kristan, 2008; Reyn et al., 2014).

One such model organism is the fruitfly larva, that will be discussed in this article. The Drosophila larva has many advantages for dissecting neural circuits, with the possibility to combine the use of precise genetic tools in Drosophila for manipulating and monitoring neuronal activity, the ongoing reconstruction of the connectome using electron microscopy, 


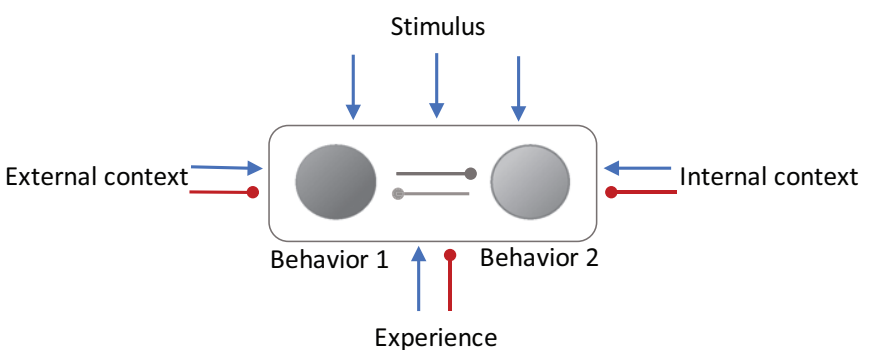

Figure 1. Schematics of flexible decisions. Sensory stimuli may evoke different behavior outputs. The different behavioral options mutually compete. These competitive interactions can be modulated by contextual information about the environment, animal's internal state and/or experience. Arrows represent excitation and lines with circles inhibition.

high-throughput assays for observing and quantifying behaviors with electrophysiology. These approaches have been used over the recent years to study the underlying mechanisms of multisensory processing (Ohyama et al., 2015), motor control (Fushiki et al., 2016; Heckscher et al., 2015; Takagi et al., 2017; Zarin, Mark, Cardona, Litwin-Kumar, \& Doe, 2019; Zwart et al., 2016), behavioral choice and sequence generation (Jovanic et al., 2016) and learning and memory (Eichler et al., 2017; Saumweber et al., 2018). While the Drosophila larva is undoubtedly a powerful model system for neural circuit dissection, the relevance of the findings from a simple nervous system to other more complex brains is often debated. This concern is two-fold: on one side, the question is whether the circuit mechanisms found in the larva will be comparable to those that take place in vertebrate nervous systems given the neuroanatomical differences. On the other, it is debated whether the decision-making process in the larva is complex enough to bring any insight that would be useful to more sophisticated forms of decision-making. These are important and valid concerns as they influence the framework for defining the problem that is being studied, interpreting the results and drawing conclusions.

The term decision-making can be ambiguous and has been used to describe processes of variable complexity across the animal kingdom from C.elegans to humans (Barker \& Baier, 2015; Constantinople, Piet, \& Brody, 2019; Faumont, Lindsay, \& Lockery, 2012; Gillette \& Brown, 2015; Glimcher \& Rustichini, 2004; Gold \& Shadlen, 2007; Hung \& Stopfer, 2018; Jarrell et al., 2012; Kristan, 2012). Generally, decisionmaking is often thought as a high-level cognitive process that leads to choices by weighing the different options and their expected outcomes often over long periods of time. In reality though, there are many different types of decisions from very simple ones like for example which hand to use to open the door, or what to order from the menu, to more complex ones such as which job to take. (Kristan, 2008; Pearson et al., 2014). In addition, many animals with very little cognition, like invertebrates, make choices of what to do (whether to eat or to escape for example) depending on both external and internal factors (Palmer \& Kristan, 2011). Some of the mechanisms underlying different aspects of these different types of decisions will be different, but others will be shared. In addition, in some cases the simple decisions have also been referred to as behavioral choice
(Faumont et al., 2012; Gaudry \& Kristan, 2009; Jovanic et al., 2016). Thus, the question of what exactly constitutes a decision has been a challenging one (Glimcher, 2004) and often the boundaries between the processes described by the terms like behavioral choice, action-selection and decisionmaking may be blurry and not always clearly distinguished.

For a purpose of this review, I will refer to decisions as events that involve a selection by the nervous system of a behavior based on various external and internal sensory information and an unambiguous commitment to that behavior (Figure 1). During decisions, the mapping from sensory inputs to motor outputs needs to be flexible for a complex and dynamic environment to be able to modulate decisions (Pearson et al., 2014). The complexity of decisions will decrease with the number of inputs and alternatives available.

I will present the case for using Drosophila larva as a model system to study the neural circuit mechanisms of decision-making. I will outline the technical advantages for dissecting neural circuits specifically from the point of view of decision-making. I will then give examples of decisions in the larva. Finally, I will propose that some of the concerns that the neural circuit mechanisms of decision-making identified in the larva may not be relevant to other species with more complex forms of decisions-making may result from the conceptual frameworks that have been traditionally used to study this problem. I argue first, that much of larval behavior has been underestimated and have been thought as hard-wired and not flexible thus not bearing a choice component. Secondly, although the complexity of decision-making of the larva may be limited compared to the type of decisions made by animals with more complex brains, some of the basic principles of how decisions are implemented in the nervous system could be shared across the animal kingdom (Adams et al., 2012). Similar computations could use similar circuit motifs as building blocks (Braganza \& Beck, 2018; Koyama \& Pujala, 2018). Thus, combining precise circuit dissection in a simple model system with investigating more complex forms of decision in complex brains could fill the gap in our knowledge about the neural circuit implementation of decision-making (Kristan, 2008).

\section{Mapping neural circuits in the Drosophila larva}

Using Drosophila larva for dissecting neural circuits has many advantages primarily due to the exquisite genetic tools that allow precise labeling of neurons and their interconnectivity as well as monitoring of neuronal activity. These precise tools can also be used to manipulate neuronal activity to determine how behaviors are affected using automated behavioral detection methods. With the help of light and more recently electron microscopy it is now possible to obtain brain-wide wiring diagrams, which opens up avenues for analysis of neural circuit at a new scale.

\subsection{Identifying elements of decision circuits}

In recent years, numerous sophisticated genetic tools that allow both the visualization and manipulation of activity of 
different cell types often at single cell resolution have been developed (Simpson, 2009; Sivanantharajah \& Zhang, 2015). These resources allow silencing/activating of neuronal activity in behaving animals and monitoring the changes that occur in their behavior. In order to quantify the changes in behaviors precisely, one needs to be able to detect behaviors unambiguously. Behavioral detection can be done either manually (Seeds et al., 2014) or automatically (AlemanMeza, Jung, \& Zhong, 2015; Gershow et al., 2012; GomezMarin, Partoune, Stephens, Louis, \& Brembs, 2012; Kabra, Robie, Rivera-Alba, Branson, \& Branson, 2013; Masson et al., 2020). The capacity to successfully identify neurons is largely increased by joining the power of genetic tools with automated analysis methods. Automatic behavioral detection allows screening libraries of neuronal lines that would be labor-costly to do using manual annotations and therefore impossible to perform at the same scale as when using automated methods (Jovanic et al., 2019; Masson et al., 2020; Robie et al., 2017; Triphan et al., 2016). In addition, automated methods allow to quantify nuanced movements as well and the precise timing of the initiation of a movement, its duration as well as the frequency of a behavior in a population of genetically identical individuals which is essential for linking neural activity to specific aspects of behavior. These tools combined with amenability of fruitflies larvae to perform large scale behavioral screens and Drosophila line libraries available have made it possible to uncover neurons relevant for particular behaviors (Kvon et al., 2014; Pfeiffer et al., 2008; 2010). This is a crucial step in mapping neural circuits, as it allows to identify its constitutive elements, whose relationships can further be tested using functional and structural connectivity studies.

To map circuits for decisions one approach is to identify a neuron that can bias decisions and then use it as a starting point to reconstruct its synaptic partners in electron microscopy (Jovanic et al., 2016; Ohyama et al., 2015). But how do we define a 'decision-making' neuron? Decision-making is a complex process that relies on the income of sensory information that is relayed by sensory pathways while their execution relies on the motor pathways. The decision outcome will thus be affected by changes in sensory processing or motor control neurons. It is therefore important to define a phenotype that we would assign to a 'decision' neuron in order to be able to discern neurons that are implicated in the decisions process from those involved in sensory processing or motor control. In an example, when multiple (two or more) options are available in response to a single sensory cue, a choice needs to made of which one to pursue or execute. When a decision is made one behavior will be selected at the expense of other behaviors. Let's take case of two behaviors $\mathrm{A}$ and $\mathrm{B}$ that are competing with each other. For each behavior, there is a neuron that is promoting that behavior and inhibiting the competing behavior. If we were to silence the activity of a neuron promoting behavior A and inhibiting behavior $\mathrm{B}$, we will expect to have a decrease of behavior A and increase of behavior B. Conversely, if we activate the same neuron, the behavior $\mathrm{A}$ will increase and behavior $\mathrm{B}$ decrease. It follows, that a decision-making phenotype would be one where the decrease in one behavior is accompanied by an increase of another or several other competing behaviors, suggesting that the neurons whose silencing results in such behavioral phenotypes, could promote one behavior and inhibit other behaviors when there are two or multiple possible options available (Masson et al., 2020).

\subsection{Mapping local circuits and brain-wide networks for decision-making}

The challenge that the nervous system puts in front of us due to the complexity of interactions between the neurons in neural networks, both local and nervous system wide, is that understanding the principles of its workings requires us to be able to interpret the function of the neurons in the context of larger neural networks.

The elegance of genetic manipulation of neural circuit elements in Drosophila larva has recently been complemented by the power of connectomics (Eichler et al., 2017; Heckscher et al., 2015; Jovanic et al., 2016; Ohyama et al., 2015; Schneider-Mizell et al., 2016; Zwart et al., 2016) as well as functional imaging methods ( $\mathrm{He}$ et al., 2019; Heckscher, Lockery, \& Doe, 2012; Karagyozov, Mihovilovic Skanata, Lesar, \& Gershow, 2018; Lemon et al., 2015; Ohyama et al., 2015; Pulver et al., 2015; Simpson \& Looger, 2018; Takagi et al., 2017; Yao, Macara, Lelito, Minosyan, \& Shafer, 2012; Zarin et al., 2019). This combination of methods has proven to be a powerful toolkit for dissecting neural circuit mechanisms.

Once a 'decision' neuron has been identified we can locate this neuron in the electron microscopy volume and reconstruct its synaptic partners (Schneider-Mizell et al., 2016). These connections can further be tested functionally for the neurons labeled in driver lines (Li et al., 2014). The implication of the reconstructed partner neurons in decision-making can further be tested using functional studies.

In order to understand the underlying mechanisms of decision-making it is not enough to look at the local circuits. There are two main views of how is the decision-making process organized in the nervous system. According to centralized models of decision-making decisions are made in specialized decision-making centers based on highly-processed sensory information that arrives there and then is relayed to the motor planning areas for execution (Miller, Galanter, \& Pribram, 1960). The non-centralized models say the decision-making process is distributed in the nervous system from the sensory to the motor side and it will be by competitive interactions between the different sensorimotor pathways that the decision will emerge (Cisek, 2007). It is therefore essential to be able to study the processes underlying decisions at the level of the entire nervous system. A compact brain such as the one of Drosophila larva can help us understand the organization of a decision-making process. In addition to mapping local circuits, in the larval brain, it is possible to trace the long-range projection neurons that connect the sites of competitions with distant regions of the nervous system. Determining the brain-wide 


\section{Crawl Stop Hunch Bend Back-up

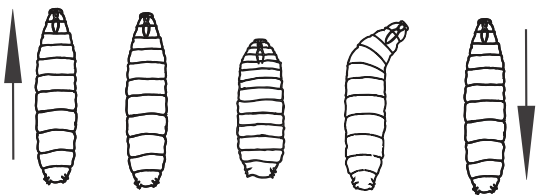

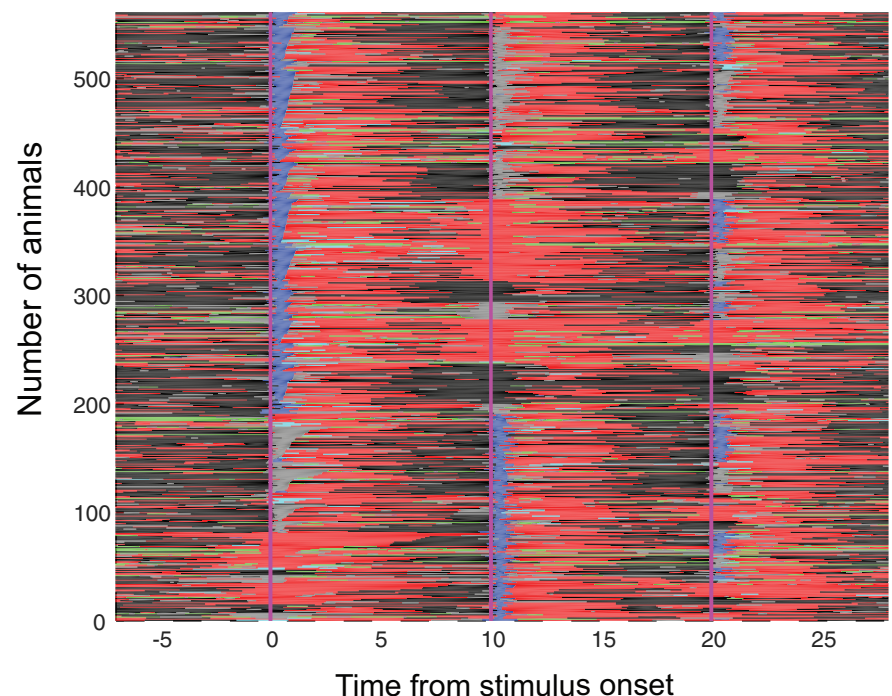

Figure 2. Ethogram of larval response to an air-puff. Each line is a larva. BlueHunch, Red-Bend, Crawl-Black, Green-Stop, Cyan-Back-up, Grey-Small motion. Different larvae respond differently. The behavioral detection used to detect the different actions is described in Masson et al. (2020). Repeated presentations of the same stimulus (here air-puff) result in different actions in the same larva.

connectivity of a 'decision' network using both structural and functional studies would help us determine whether decision-making is distributed or centralized and to which extent.

\section{What decisions do Drosophila larvae make?}

For all of these reasons, namely the precise labeling and manipulation of single neurons to brain-wide maps of neural network, Drosophila is powerful system to dissect neural circuits. But how relevant is it to understand the neural circuit structure and function underlying decisions in a small insect brain? What types of decisions do they make and is the nature of the underlying processes in these behaviors similar to the ones occurring during more complex decisions in more complex brains?

Generally, in its simplest form, a decision is the choice of the type of behavior to execute in response external and/or internal sensory information when there are multiple possibilities available. This can be a choice between to respond or not to respond or a choice between different types of actions (Gold \& Shadlen, 2007). What will make decisions more or less complex will be the type and features of the sensory information, on one side and on the other, the number and types of behavioral options available (and the level of certainty about their respected outcomes) (Schleyer, Diegelmann, Michels, Saumweber, \& Gerber, 2013). Finally, decisions will be modulated by the external context, larva's internal, motivational, behavioral state and experience.
Drosophila larvae have innate preferences for some stimuli and can be repelled by others. For instance, in presence of appetitive odors the larvae will approach the source of odor (Fishilevich et al., 2005; Gomez-Marin, Stephens, \& Louis, 2011; Louis, Huber, Benton, Sakmar, \& Vosshall, 2008). In presence of aversive or noxious stimuli they will use strategies to escape or avoid the unpleasant situation. This is true for examples, for responses to light that increase head-casting (Zhao et al., 2017), high temperature that increases crawling speed, mechanosensory stimuli: vibration, air-puff, touch that induce hunching, bending, stopping and crawling backwards and in some cases, reorientation (Jovanic et al., 2016; Ohyama et al., 2013; Tsubouchi, Caldwell, \& Tracey, 2012; Yan et al., 2013; Zhang, Yan, Jan, \& Jan, 2013) or noxious stimuli that can induce rolling (Burgos et al., 2018; Ohyama et al., 2015; Robertson, Tsubouchi, \& Tracey, 2013) or full body contraction (Turner et al., 2016). Thus, larvae respond to different types of stimuli with different actions. But even to a single stimulus they can respond differently especially if these stimuli are weak or ambiguous (Jovanic et al., 2016; Tsubouchi et al., 2012). The same stimulus may evoke different behaviors in different larvae or upon repeated presentation in the same larva (Jovanic et al., 2016) (Figure 2). Thus, Drosophila larvae have a rich repertoire of flexible sensorimotor behaviors. The sensory neurons mediating these responses have been well characterized (Klein et al., 2015; Merritt \& Whitington, 1995; Sprecher, Cardona, \& Hartenstein, 2011; Xiang et al., 2010) and the downstream neural circuitry have started to be unraveled, revealing multilevel convergence architecture for enhanced action selection during responses to multimodal stimuli (Ohyama et al., 2015), disinhibitory mechanisms of behavioral choice (Jovanic et al., 2016; Zhao et al., 2019), modular organization of escape behaviors (Burgos et al., 2018) and a bistable inhibitory system for olfactory processing among others.

Many of the behaviors of Drosophila larvae can be thought of as approaching good things (appetitive behaviors) and avoiding bad things (aversive behaviors). Larvae orient themselves in different types of sensory gradients (Gepner, Mihovilovic Skanata, Bernat, Kaplow, \& Gershow, 2015; Gershow et al., 2012; Gomez-Marin et al., 2011; Jovanic et al., 2019; Kane et al., 2013; Luo et al., 2010; Schulze et al., 2015; Tastekin et al., 2018; 2015) and they will use different locomotor strategies to move in space up or down the gradients. In gradients of different modalities larvae use similar strategies: they modulate runs and turns (frequency and direction) to move away from unfavorable conditions and towards favorable conditions. Larvae performs these navigational decisions during chemotaxis (in odor gradients), phototaxis (light intensity gradients), thermotaxis (temperature gradients), anemotaxis (wind speed gradients), gradients of $\mathrm{CO}_{2}$ and in combination of multiple possibly conflicting sensory gradients (Gepner et al., 2015). Sensory neurons involved in sensing these gradients have by large been described and central neurons have started to be identified by manipulating their activity during various taxes (Gong et al., 2010; Kane et al., 2013; Slater, Levy, Chan, \& Larsen, 
2015; Tastekin et al., 2015; 2018). It will interesting to correlate the activity of these neurons to specific navigational strategies in behaving larvae (Karagyozov et al., 2018) to better understand their role in navigational decision-making.

\subsection{Modulation of decisions by the internal state}

Animal's internal physiological state will affect animal's receptivity to its environment (Palmer \& Kristan, 2011). For example, an animal that is hungry might ignore aversive external stimuli as it will be primarily motivated by searching for food and will thus continue foraging. A type of decision that will strongly be influenced by internal state are decisions related to feeding. When faced with the option to feed, several different decisions must be made: first whether or not to eat, what and how much to eat, and when to eat (Bjordal, Arquier, Kniazeff, Pin, \& Léopold, 2014; Itskov \& Ribeiro, 2013; Kudow et al., 2017). In order to make appropriate choices the nervous system has to evaluate internal energy (nutrients) level and environmental information (presence of food or dangers) to make decisions whether, when and how to feed. The latter is achieved through a sophisticated olfactory and gustatory systems that allow to search and detect different types of food sources (Schleyer et al., 2013). Satiation state influences the decisions what to eat (Itskov \& Ribeiro, 2013) and olfactory and gustatory preferences are modified in hungry larvae. For example, normally Drosophila larvae exposed to pathogenic bacteria move away from the food. However, this evasion behavior was diminished in starved larvae that overcome the aversions of danger to look for food (Surendran, Hückesfeld, Wäschle, \& Pankratz, 2017). Recently sensory neurons for sensing of the internals state in the larva have been described proving an entry point into elucidate molecular and circuit mechanisms underlying sensory processing of internal body state and how it affects behavioral choice (Qian, Kaplow, Lee, \& Grueber, 2018). These decisions will be further modulated by other competing internal or external stimuli. Indeed larval food search behavior that involves risky diving into hydrogel illustrates larval decisions that balance risk against benefit (Kim, Alvarez, Lechuga, \& Louis, 2017).

\subsection{Contextual modulation of decisions}

Decision can be modulated by the multisensory context. The presence of stimuli from multiple modalities that inform about the same object (i.e. a parasitoid wasp) will enhance action selection in the Drosophila larva (Ohyama et al., 2015). Sometimes information from different sensory modalities can provide conflicting alternatives and the larva will need to make a choice between the two (Koseki et al., 2016). Mechanisms must exist that ensure that the different circuits interact to make an adaptive choice.

Finally, the environmental context can also modulate decisions by modifying the behavioral options available (Cisek, 2007; Cisek \& Kalaska, 2010). Different environments can provide different opportunities for expressing different behaviors. For example, in response to a an air-puff larvae will typically perform different avoidance action types on the surface of the agar plate (Figure 2) (Jovanic et al., 2016; Masson et al., 2020; Ohyama et al., 2013). However, if in the agar surface we introduce small holes, larvae will dig into the agar in presence of air-puff more than in its absence (unpublished). The affordance provided by the holes as a potential place where the larva could dig into to protect itself from the wind changed larval behavior in response to an air-puff stimulus. Thus, decisions can be modulated by the environmental context in terms for opportunities for actions it provides.

\subsection{Modulation of decision by the experience}

Interactions between the animal and environment will result in memories that can be called to inform future decisions. Through this process larvae will learn that some cues might be associated with reward or punishment and will express this memory in the case it is useful for improving their situation (Schleyer et al., 2013; Schleyer, Fendt, Schuller, \& Gerber, 2018). The decision to behaviorally express an associative memory trace and the architecture of the chemobehavioral system have been reviewed in (Schleyer et al., 2013). The connectome of the mushroom body, learning and memory center, in the larva has been reconstructed (Eichler et al., 2017) and its functional architecture characterized (Saumweber et al., 2018) which provides the basis for detailed functional studies of conditioned behavior (i.e. Eschbach et al. (2019)).

\section{Relevance of studying decision-making in the drosophila larva}

While the stimulus-response model has often been used to describe behaviors in simple model systems and thus defined them as hard-wired, lacking flexibility and not resulting from animals' choice but rather being an automated response to a stimulus, recent studies show that even the simplest behaviors may possess behavioral plasticity (Gorostiza, 2018) allowing the animal to modify their behavior responses according to environmental context, metabolic demands and physiological state.

Another important consideration is the fact that in the context of a lab, behavioral variability is controlled in order to be able to observe, measure and quantify the behavior or aspects of behaviors of interest and link it to underlying circuit mechanisms. Although this simplification is necessary to make causal links between circuit elements and specific aspects of decisions, it needs to be taken into account as this may make the behaviors seem more rigid and less plastic than they might actually be (Gorostiza, 2018). For example, typically it is necessary to devise paradigms that allow us to specifically reveal learning and memory effects on behavior and its underlying mechanisms. However, given the Drosophila larvae nervous system possesses the circuitry and molecular machinery to learn, its behavior might be shaped by prior exposure to similar stimuli information even in behavioral paradigm that are dominated by innate behaviors. 
While it is obvious that the decision-making process in the Drosophila larva lacks the cognitive and emotional complexity of the one in us humans, or other vertebrates, I believe it is fair to acknowledge that the various examples of larval Drosophila behaviors from the previous sections illustrate decision-making of various complexity: whether it is to select different types of escape responses (Jovanic et al., 2016; Ohyama et al., 2015) or to make cost benefit decisions (Kim et al., 2017). These rather simple decisions share some of the features of the decision-making problem in general. For example, even in the simplest cases of decision-making, there will be competing demands on the effector systems and therefore an organization in the nervous system needs to be in place to successfully and accurately resolve these conflicts. In addition, the mapping between sensory information and motor output needs to be flexible to enable the multisensory context, state and experience to all modulate decisions (Figure 1).

The principles of how competitive interactions of flexible decisions are implemented at the level of neural circuits may thus be studied in the larva, but how likely it is that the neural architectures for decision-making in the larva will be relevant to decisions in more complex vertebrate brains? Connectivity patterns that give rise to particular neuronal dynamics have been shown to appear in multiple nervous systems. One such motif is mutual inhibition of inhibition found in multiple species to implement competitive interactions and similar computations (Goddard, Mysore, Bryant, Huguenard, \& Knudsen, 2014; Jovanic et al., 2016; Koyama et al., 2016; Koyama \& Pujala, 2018). This concept of circuit motifs as building blocks of elementary computations that are reused to implement similar functions across brain areas and nervous systems (Koyama \& Pujala, 2018) offers a level of abstraction that can be used to extract the principles of circuit function. These can be further applied to many different nervous systems. In this view, understanding the detailed mechanisms of how choice is made between conflicting drives and how the constantly incoming information about the environment and the internal state feed into this process, regardless of the complexity of the selection problem, may provide a solid principled knowledge about decision-making and open new avenues, establish new concepts and offer a new interpretation of existing experimental data (Kristan, 2008).

Over the recent years a view has emerged that decisions are made through competitive interactions of sensorimotor pathways. In the view of this interactive model of decisionmaking, simultaneous processes of sensorimotor control and action selection are distributed in the nervous system (Alcaraz et al., 2015; Cisek, 2007; Cisek \& Kalaska, 2010). It is thus essential to be able to study its neural implementation in a brain-wide manner. Understanding decision-making in a compact brain like the one of the fruitfly larva where such endeavor is feasible with cellular and synaptic resolution, can offer a roadmap for elucidating the mechanisms in more complex brains by giving us clues where to look and what kind of structures to expect. Compared to the classical view of decision-making as a series of processing stages, the interactive framework sheds light on decisions in a new way. While this framework may not apply to all decisions equally, it may allow us to bridge the gap between the view that invertebrate behaviors are hard-wired innate routines, each being triggered by a combination of external stimuli and internal drivers, while more complex organisms go a through deliberate decision-making composed of several stages of computation before taking any external actions.

\section{Acknowledgements}

The author thanks Daniel Vasiliauskas for comments on this manuscript.

\section{Disclosure statement}

No potential conflict of interest was reported by the authors.

\section{Funding}

This project has received funding from the European Union's Horizon 2020 research and innovation programme under the Marie Sklodowska-Curie grant agreement No 798050 and from the ANR (agence nationale de la recherche): ANR-17-CE37-0019.

\section{ORCID}

Tihana Jovanic (D) http://orcid.org/0000-0002-0525-8620

\section{References}

Adams, G.K., Watson, K.K., Pearson, J., \& Platt, M.L. (2012). Neuroethology of decision-making. Current Opinion in Neurobiology, 22, 982-989. doi:10.1016/j.conb.2012.07.009

Alcaraz, F., Marchand, A.R., Vidal, E., Guillou, A., Faugère, A., Coutureau, E., \& Wolff, M. (2015). Flexible use of predictive cues beyond the orbitofrontal cortex: role of the submedius thalamic nucleus. The Journal of Neuroscience: The Official Journal of the Society for Neuroscience, 35, 13183-13193. doi:10.1523/JNEUROSCI. 1237-15.2015

Aleman-Meza, B., Jung, S.-K., \& Zhong, W. (2015). An automated system for quantitative analysis of Drosophila larval locomotion. BMC Developmental Biology, 15, 11. doi:10.1186/s12861-015-0062-0

Baca, S.M., Marin-Burgin, A., Wagenaar, D.A., \& Kristan, W.B. Jr. (2008). Widespread inhibition proportional to excitation controls the gain of a leech behavioral circuit. Neuron, 57, 276-289. doi:10. 1016/j.neuron.2007.11.028

Barker, A.J., \& Baier, H. (2015). Sensorimotor decision making in the zebrafish tectum. Current Biology, 25, 2804-2814. doi:10.1016/j.cub. 2015.09.055

Bjordal, M., Arquier, N., Kniazeff, J., Pin, J.P., \& Léopold, P. (2014). Sensing of amino acids in a dopaminergic circuitry promotes rejection of an incomplete diet in Drosophila. Cell, 156, 510-521. doi:10. 1016/j.cell.2013.12.024

Braganza, O., \& Beck, H. (2018). The circuit motif as a conceptual tool for multilevel neuroscience. Trends in Neurosciences, 41, 128-136. doi:10.1016/j.tins.2018.01.002

Burgos, A., Honjo, K., Ohyama, T., Qian, C.S., Shin, G. J-e., Gohl, D.M., ... Grueber, W.B. (2018). Nociceptive interneurons control modular motor pathways to promote escape behavior in Drosophila. eLife, 7, 1557. doi:10.7554/eLife.26016 
Cisek, P. (2007). Cortical mechanisms of action selection: the affordance competition hypothesis. Philosophical Transactions of the Royal Society B: Biological Sciences, 362, 1585-1599. doi:10.1098/rstb.2007. 2054

Cisek, P., \& Kalaska, J.F. (2010). Neural Mechanisms for Interacting with a World Full of Action Choices. Annual Review of Neuroscience, 33, 269-298. doi:10.1146/annurev.neuro.051508.135409

Constantinople, C.M., Piet, A.T., \& Brody, C.D. (2019). An analysis of decision under risk in rats. Current Biology, 29, 2066-2074.e5. doi: 10.1016/j.cub.2019.05.013

Eichler, K., Li, F., Litwin-Kumar, A., Park, Y., Andrade, I., SchneiderMizell, C.M., ... Cardona, A. (2017). The complete connectome of a learning and memory centre in an insect brain. Nature, 548, 175-182. doi:10.1038/nature23455

Eschbach, C., Fushiki, A., Winding, M., Schneider-Mizell, C.M., Shao, M., \& Arruda, R. (2019). Multilevel feedback architecture for adaptive regulation of learning in the insect brain. bioRxiv, 13, 1557-1544. doi:10.1101/649731

Faumont, S., Lindsay, T.H., \& Lockery, S.R. (2012). Neuronal microcircuits for decision making in C. elegans. Current Opinion in Neurobiology, 22, 580-591. doi:10.1016/j.conb.2012.05.005

Fishilevich, E., Domingos, A.I., Asahina, K., Naef, F., Vosshall, L.B., \& Louis, M. (2005). Chemotaxis behavior mediated by single larval olfactory neurons in Drosophila. Curbio, 15, 2086-2096. doi:10.1016/ j.cub.2005.11.016

Fushiki, A., Zwart, M.F., Kohsaka, H., Fetter, R.D., Cardona, A., Nose, A., \& Griffith, L.C. (2016). A circuit mechanism for the propagation of waves of muscle contraction in Drosophila. eLife, 5, e13253. doi: 10.7554/eLife. 13253

Gaudry, Q., \& Kristan, W.B. (2009). Behavioral choice by presynaptic inhibition of tactile sensory terminals. Nature Publishing Group, 12, 1450-1457. doi:10.1038/nn.2400

Gepner, R., Mihovilovic Skanata, M., Bernat, N.M., Kaplow, M., \& Gershow, M. (2015). Computations underlying Drosophila phototaxis, odor-taxis, and multi-sensory integration. eLife, 4, e06229. doi: 10.7554/eLife.06229

Gershow, M., Berck, M., Mathew, D., Luo, L., Kane, E.A., Carlson, J.R., \& Samuel, A.D.T. (2012). Controlling airborne cues to study small animal navigation. Nature Methods, 9, 290-296. doi:10.1038/nmeth. 1853

Gillette, R., \& Brown, J.W. (2015). The sea slug, Pleurobranchaea californica: a signpost species in the evolution of complex nervous systems and behavior. Integrative and Comparative Biology, 55, 1058-1069.

Glimcher, P.W. (2004). Decisions, uncertainty, and the brain. Cambridge, MA: MIT Press.

Glimcher, P.W., \& Rustichini, A. (2004). Neuroeconomics: the consilience of brain and decision. Science, 306, 447-452. doi:10.1126/science. 1102566

Goddard, C.A., Mysore, S.P., Bryant, A.S., Huguenard, J.R., \& Knudsen, E.I. (2014). Spatially reciprocal inhibition of inhibition within a stimulus selection network in the avian midbrain. PloS One, 9, e85865. doi:10.1371/journal.pone.0085865

Gold, J.I., \& Shadlen, M.N. (2007). The neural basis of decision making. Annual Review of Neuroscience, 30, 535-574. doi:10.1146/ annurev.neuro.29.051605.113038

Gomez-Marin, A., Partoune, N., Stephens, G.J., Louis, M., \& Brembs, B. (2012). Automated tracking of animal posture and movement during exploration and sensory orientation behaviors. PloS One, 7, e41642. doi:10.1371/journal.pone.0041642

Gomez-Marin, A., Stephens, G.J., \& Louis, M. (2011). Active sampling and decision making in Drosophila chemotaxis. Nature Communications, 2, 441. doi:10.1038/ncomms1455

Gong, Z., Liu, J., Guo, C., Zhou, Y., Teng, Y., \& Liu, L. (2010). Two pairs of neurons in the central brain control Drosophila innate light preference. Science, 330, 499-502. doi:10.1126/science.1195993

Gorostiza, E.A. (2018). Does cognition have a role in plasticity of "innate behavior?" a perspective from Drosophila. Frontiers in Psychology, 9, 1502. doi:10.3389/fpsyg.2018.01502
He, L., Gulyanon, S., Mihovilovic Skanata, M., Karagyozov, D., Heckscher, E.S., Krieg, M., ... Tracey, W.D. (2019). Direction selectivity in Drosophila proprioceptors requires the mechanosensory channel Tmc. Current Biology, 29, 945-956.e3. doi:10.1016/j.cub. 2019.02.025

Heckscher, E.S., Lockery, S.R., \& Doe, C.Q. (2012). Characterization of Drosophila larval crawling at the level of organism, segment, and somatic body wall musculature. The Journal of Neuroscience: The Official Journal of the Society for Neuroscience, 32, 12460-12471. doi: 10.1523/JNEUROSCI.0222-12.2012

Heckscher, E.S., Zarin, A.A., Faumont, S., Clark, M.Q., Manning, L., Fushiki, A., ... Doe, C.Q. (2015). Even-skipped(+) interneurons are core components of a sensorimotor circuit that maintains left-right symmetric muscle contraction amplitude. Neuron, 88, 314-329. doi: 10.1016/j.neuron.2015.09.009

Hung, Y.-S., \& Stopfer, M. (2018). Decision making: how fruit flies integrate olfactory evidence. Current Biology, 28, R757-R759. doi:10. 1016/j.cub.2018.05.065

Itskov, P.M., \& Ribeiro, C. (2013). The dilemmas of the gourmet fly: the molecular and neuronal mechanisms of feeding and nutrient decision making in Drosophila. Frontiers in Neuroscience, 7, 12. doi: 10.3389/fnins.2013.00012

Jarrell, T.A., Wang, Y., Bloniarz, A.E., Brittin, C.A., Xu, M., Thomson, J.N., ... Emmons, S.W. (2012). The connectome of a decision-making neural network. Science, 337, 437-444. doi:10.1126/science. 1221762

Jovanic, T., Schneider-Mizell, C.M., Shao, M., Masson, J.-B., Denisov, G., Fetter, R.D., ... Zlatic, M. (2016). Competitive disinhibition mediates behavioral choice and sequences in Drosophila. Cell, 167, 858-870.e19. doi:10.1016/j.cell.2016.09.009

Jovanic, T., Winding, M., Cardona, A., Truman, J.W., Gershow, M., \& Zlatic, M. (2019). Neural substrates of Drosophila larval anemotaxis. Current Biology, 29, 554-566.e4. doi:10.1016/j.cub.2019.01.009

Kabra, M., Robie, A.A., Rivera-Alba, M., Branson, S., \& Branson, K. (2013). JAABA: interactive machine learning for automatic annotation of animal behavior. Nature Methods, 10, 64-67. doi:10.1038/ nmeth. 2281

Kane, E.A., Gershow, M., Afonso, B., Larderet, I., Klein, M., Carter, A.R., ... Samuel, A.D.T. (2013). Sensorimotor structure of Drosophila larva phototaxis. Proceedings of the National Academy of Sciences of the United States of America, 110, E3868-77. doi:10.1073/ pnas. 1215295110

Karagyozov, D., Mihovilovic Skanata, M., Lesar, A., \& Gershow, M. (2018). Recording neural activity in unrestrained animals with threedimensional tracking two-photon microscopy. Cell Reports, 25, 1371-1383.e10. doi:10.1016/j.celrep.2018.10.013

Kim, D., Alvarez, M., Lechuga, L.M., \& Louis, M. (2017). Species-specific modulation of food-search behavior by respiration and chemosensation in Drosophila larvae. eLife, 6, 356. doi:10.7554/eLife.27057

Klein, M., Afonso, B., Vonner, A.J., Hernandez-Nunez, L., Berck, M., Tabone, C.J., ... Samuel, A.D.T. (2015). Sensory determinants of behavioral dynamics in Drosophila thermotaxis. Proceedings of the National Academy of Sciences of the United States of America, 112, E220-9. doi:10.1073/pnas.1416212112

Koseki, N., Mori, S., Suzuki, S., Tonooka, Y., Kosugi, S., Miyakawa, H., \& Morimoto, T. (2016). Individual differences in sensory responses influence decision making by Drosophila melanogaster larvae on exposure to contradictory cues. Journal of Neurogenetics, 30, 288-296. doi:10.1080/01677063.2016.1202949

Kovac, M.P., \& Davis, W.J. (1977). Behavioral choice: neural mechanisms in Pleurobranchaea. Science, 198, 632-634. doi:10.1126/science.918659

Koyama, M., Minale, F., Shum, J., Nishimura, N., Schaffer, C.B., \& Fetcho, J.R. (2016). A circuit motif in the zebrafish hindbrain for a two alternative behavioral choice to turn left or right. eLife, 5, 451. doi:10.7554/eLife. 16808

Koyama, M., \& Pujala, A. (2018). Mutual inhibition of lateral inhibition: a network motif for an elementary computation in the brain. Current Opinion in Neurobiology, 49, 69-74. doi:10.1016/j.conb.2017. 12.019 
Kristan, W.B. (2008). Neuronal decision-making circuits. Current Biology, 18, R928-R932. doi:10.1016/j.cub.2008.07.081

Kristan, W.B. Jr. (2012). Decision points: the factors influencing the decision to feed in the medicinal leech. Frontiers in Neuroscience, 6, 101. doi: $10.3389 /$ fnins.2012.00101/abstract

Kudow, N., Miura, D., Schleyer, M., Toshima, N., Gerber, B., \& Tanimura, T. (2017). Preference for and learning of amino acids in larval Drosophila. Biology Open, 6, 365-369. doi:10.1242/bio.020412

Kupfermann, I., \& Weiss, K.R. (2001). Motor program selection in simple model systems. Current Opinion in Neurobiology, 11, 673-677. doi:10.1016/S0959-4388(01)00267-7

Kvon, E.Z., Kazmar, T., Stampfel, G., Yáñez-Cuna, J.O., Pagani, M., Schernhuber, K., ... Stark, A. (2014). Genome-scale functional characterization of Drosophila developmental enhancers in vivo. Nature, 512, 91-95. doi:10.1038/nature 13395

Lemon, W.C., Pulver, S.R., Höckendorf, B., McDole, K., Branson, K., Freeman, J., \& Keller, P.J. (2015). Whole-central nervous system functional imaging in larval Drosophila. Nature Communications, 6, 7924-7916. doi:10.1038/ncomms8924

Li, H.-H., Kroll, J.R., Lennox, S.M., Ogundeyi, O., Jeter, J., Depasquale, G., \& Truman, J.W. (2014). A GAL4 driver resource for developmental and behavioral studies on the larval CNS of Drosophila. Cell Reports, 8, 897-908. doi:10.1016/j.celrep.2014.06.065

Louis, M., Huber, T., Benton, R., Sakmar, T.P., \& Vosshall, L.B. (2008). Bilateral olfactory sensory input enhances chemotaxis behavior. Nature Neuroscience, 11, 187-199. doi:10.1038/nn2031

Luo, L., Gershow, M., Rosenzweig, M., Kang, K., Fang-Yen, C., Garrity, P.A., \& Samuel, A.D.T. (2010). Navigational decision making in Drosophila thermotaxis. The Journal of Neuroscience: The Official Journal of the Society for Neuroscience, 30, 4261-4272. doi:10.1523/ JNEUROSCI.4090-09.2010

Maass, W. (2000). On the computational power of winner-take-all. Neural Computation, 12, 2519-2535. doi:10.1162/089976600300014827

Masson, J.-B., Laurent, F., Cardona, A., Barre, C., Skatchkovsky, N., \& Truman, J.W. (2020). Identifying neural substrates of competitive interactions and sequence transitions during mechanosensory responses in Drosophila. Plos Genetics. (in press)

Merritt, D.J., \& Whitington, P.M. (1995). Central projections of sensory neurons in the Drosophila embryo correlate with sensory modality, soma position, and proneural gene function. The Journal of Neuroscience: The Official Journal of the Society for Neuroscience, 15, 1755-1767. doi:10.1523/JNEUROSCI.15-03-01755.1995

Miller, G.A., Galanter, E., \& Pribram, K.H. (1960). Plans and the structure of behavior. New York: Holt, Rinehart and Winston, Inc.

Mink, J.W. (1996). The basal ganglia: focused selection and inhibition of competing motor programs. Progress in Neurobiology, 50, 381-425. doi:10.1016/S0301-0082(96)00042-1

Ohyama, T., Jovanic, T., Denisov, G., Dang, T.C., Hoffmann, D., Kerr, R.A., \& Zlatic, M. (2013). High-throughput analysis of stimulusevoked behaviors in Drosophila larva reveals multiple modality-specific escape strategies. PloS One, 8, e71706. doi:10.1371/journal.pone. 0071706

Ohyama, T., Schneider-Mizell, C.M., Fetter, R.D., Aleman, J.V., Franconville, R., Rivera-Alba, M., ... Zlatic, M. (2015). A multilevel multimodal circuit enhances action selection in Drosophila. Nature, 520, 633-639. doi:10.1038/nature14297

Palmer, C.R., \& Kristan, W.B. (2011). Contextual modulation of behavioral choice. Current Opinion in Neurobiology, 21, 520-526. doi:10. 1016/j.conb.2011.05.003

Pearson, J.M., Watson, K.K., \& Platt, M.L. (2014). Decision making: the neuroethological turn. Neuron, 82, 950-965. doi:10.1016/j.neuron.2014.04.037

Pfeiffer, B.D., Jenett, A., Hammonds, A.S., Ngo, T.-T.B., Misra, S., Murphy, C., ... Rubin, G.M. (2008). Tools for neuroanatomy and neurogenetics in Drosophila. Proceedings of the National Academy of Sciences of the United States of America, 105, 9715-9720. doi:10. 1073/pnas.0803697105

Pfeiffer, B.D., Ngo, T.-T.B., Hibbard, K.L., Murphy, C., Jenett, A., Truman, J.W., \& Rubin, G.M. (2010). Refinement of tools for targeted gene expression in Drosophila. Genetics, 186, 735-755. doi: 10.1534/genetics.110.119917

Prescott, T.J. (2007). Forced moves or good tricks in design space?Landmarks in the evolution of neural mechanisms for action selection. Adaptive Behavior, 15, 9-31. doi:10.1177/ 1059712306076252

Pulver, S.R., Bayley, T.G., Taylor, A.L., Berni, J., Bate, M., \& Hedwig, B. (2015). Imaging fictive locomotor patterns in larval Drosophila. Journal of Neurophysiology, 114, 2564-2577. doi:10.1152/jn.00731. 2015

Qian, C.S., Kaplow, M., Lee, J.K., \& Grueber, W.B. (2018). Diversity of internal sensory neuron axon projection patterns is controlled by the POU-domain protein Pdm3 in Drosophila larvae. The Journal of Neuroscience: The Official Journal of the Society for Neuroscience, 38, 2081-2093. doi:10.1523/JNEUROSCI.2125-17.2018

Redgrave, P., Prescott, T.J., \& Gurney, K. (1999). The basal ganglia: a vertebrate solution to the selection problem? Neuroscience, 89, 1009-1023. doi:10.1016/S0306-4522(98)00319-4

Redgrave, P., Vautrelle, N., \& Reynolds, J.N.J. (2011). Functional properties of the basal ganglia's re-entrant loop architecture: selection and reinforcement. Nsc, 198, 138-151. doi:10.1016/j.neuroscience. 2011.07.060

Reyn, V. C.R., Breads, P., Peek, M.Y., Zheng, G.Z., Williamson, W.R., \& Yee, A.L. (2014). A spike-timing mechanism for action selection. Nature Neuroscience, 17, 962-970. doi:10.1038/nn.3741

Robertson, J.L., Tsubouchi, A., \& Tracey, W.D. (2013). Larval defense against attack from parasitoid wasps requires nociceptive neurons. PloS One, 8, e78704. doi:10.1371/journal.pone.0078704

Robie, A.A., Hirokawa, J., Edwards, A.W., Umayam, L.A., Lee, A., Phillips, M.L., ... Branson, K. (2017). Mapping the neural substrates of behavior. Cell, 170, 393-406.e28. doi:10.1016/j.cell.2017.06.032

Saumweber, T., Rohwedder, A., Schleyer, M., Eichler, K., Chen, Y-c., Aso, Y., ... Gerber, B. (2018). Functional architecture of reward learning in mushroom body extrinsic neurons of larval Drosophila. Nature Communications, 9, 1104. doi:10.1038/s41467-018-03130-1

Schleyer, M., Diegelmann, S., Michels, B., Saumweber, T., \& Gerber, B. (2013). Decision making in larval Drosophila. Handbook of Behavioral Neuroscience, 22, 41-55. doi:10.1016/B978-0-12-415823-8. 00005-8

Schleyer, M., Fendt, M., Schuller, S., \& Gerber, B. (2018). Associative learning of stimuli paired and unpaired with reinforcement: evaluating evidence from maggots, flies, bees, and rats. Frontiers in Psychology, 9, 1494. doi:10.3389/fpsyg.2018.01494

Schneider-Mizell, C.M., Gerhard, S., Longair, M., Kazimiers, T., Li, F., Zwart, M.F., ... Cardona, A. (2016). Quantitative neuroanatomy for connectomics in Drosophila. eLife, 5, 1133. doi:10.7554/eLife.12059

Schulze, A., Gomez-Marin, A., Rajendran, V.G., Lott, G., Musy, M., Ahammad, P., ... Louis, M. (2015). Dynamical feature extraction at the sensory periphery guides chemotaxis. eLife, 4, 1129. doi:10.7554/ eLife.06694

Seeds, A.M., Ravbar, P., Chung, P., Hampel, S., Midgley, F.M., Mensh, B.D., \& Simpson, J.H. (2014). A suppression hierarchy among competing motor programs drives sequential grooming in Drosophila. eLife 3, e02951.

Simpson, J.H. (2009). Mapping and manipulating neural circuits in the fly brain. Advances in Genetics, 65, 79-143. doi:10.1016/S00652660(09)65003-3

Simpson, J.H., \& Looger, L.L. (2018). Functional imaging and optogenetics in Drosophila. Genetics, 208, 1291-1309. doi:10.1534/genetics. 117.300228

Sivanantharajah, L., \& Zhang, B. (2015). Current techniques for highresolution mapping of behavioral circuits in Drosophila. Journal of Comparative Physiology. a, Neuroethology, Sensory, Neural, and Behavioral Physiology, 201, 895-909. doi:10.1007/s00359-015-1010-y

Slater, G., Levy, P., Chan, K.L.A., \& Larsen, C. (2015). A central neural pathway controlling odor tracking in Drosophila. The Journal of Neuroscience: The Official Journal of the Society for Neuroscience, 35, 1831-1848. doi:10.1523/JNEUROSCI.2331-14.2015

Sprecher, S.G., Cardona, A., \& Hartenstein, V. (2011). The Drosophila larval visual system: high-resolution analysis of a simple visual 
neuropil. Developmental Biology, 358, 33-43. doi:10.1016/j.ydbio. 2011.07.006

Surendran, S., Hückesfeld, S., Wäschle, B., \& Pankratz, M.J. (2017). Pathogen-induced food evasion behavior in Drosophila larvae. The Journal of Experimental Biology, 220, 1774-1780. doi:10.1242/jeb. 153395

Takagi, S., Cocanougher, B.T., Niki, S., Miyamoto, D., Kohsaka, H., Kazama, H., ... Nose, A. (2017). Divergent connectivity of homologous command-like neurons mediates segment-specific touch responses in Drosophila. Neuron, 96, 1373-1387.e6. doi:10.1016/j. neuron.2017.10.030

Tastekin, I., Khandelwal, A., Tadres, D., Fessner, N.D., Truman, J.W., Zlatic, M., ... Louis, M. (2018). Sensorimotor pathway controlling stopping behavior during chemotaxis in the Drosophila melanogaster larva. eLife, 7, 2452. doi:10.7554/eLife.38740

Tastekin, I., Riedl, J., Schilling-Kurz, V., Gomez-Marin, A., Truman, J.W., \& Louis, M. (2015). Role of the subesophageal zone in sensorimotor control of orientation in Drosophila larva. Current Biology, 25, 1448-1460. doi:10.1016/j.cub.2015.04.016

Triphan, T., Nern, A., Roberts, S.F., Korff, W., Naiman, D.Q., \& Strauss, R. (2016). A screen for constituents of motor control and decision making in Drosophila reveals visual distance-estimation neurons. Scientific Reports, 6. Article number: 27000. doi:10.1038/srep27000

Tsubouchi, A., Caldwell, J.C., \& Tracey, W.D. (2012). Dendritic filopodia, ripped pocket, NOMPC, and NMDARs contribute to the sense of touch in Drosophila larvae. Current Biology, 22, 2124-2134. doi: 10.1016/j.cub.2012.09.019

Turner, H.N., Armengol, K., Patel, A.A., Himmel, N.J., Sullivan, L., Iyer, S.C., ... Cox, D.N., et al. (2016). The TRP channels Pkd2, NompC, and Trpm act in cold-sensing neurons to mediate unique aversive behaviors to noxious cold in Drosophila. Current Biology, 26, 3116-3128. doi:10.1016/j.cub.2016.09.038

Wang, X.-J. (2008). Decision making in recurrent neuronal circuits. Neuron, 60, 215-234. doi:10.1016/j.neuron.2008.09.034
Xiang, Y., Yuan, Q., Vogt, N., Looger, L.L., Jan, L.Y., \& Jan, Y.-N. (2010). Light-avoidance-mediating photoreceptors tile the Drosophila larval body wall. Nature, 468, 921-926. doi:10.1038/nature09576

Yan, Z., Zhang, W., He, Y., Gorczyca, D., Xiang, Y., Cheng, L.E., ... Jan, Y.N., et al. (2013). Drosophila NOMPC is a mechanotransduction channel subunit for gentle-touch sensation. Nature, 493, 221-225. doi:10.1038/nature11685

Yao, Z., Macara, A.M., Lelito, K.R., Minosyan, T.Y., \& Shafer, O.T. (2012). Analysis of functional neuronal connectivity in the Drosophila brain. Journal of Neurophysiology, 108, 684-696. doi:10. 1152/jn.00110.2012

Zarin, A.A., Mark, B., Cardona, A., Litwin-Kumar, A., \& Doe, C.Q. (2019b). A multilayer circuit architecture for the generation of distinct locomotor behaviors in Drosophila. eLife, 8, e51781. doi:10. 7554/eLife.51781

Zhang, W., Yan, Z., Jan, L.Y., \& Jan, Y.-N. (2013). Sound response mediated by the TRP channels NOMPC, NANCHUNG, and INACTIVE in chordotonal organs of Drosophila larvae. Proceedings of the National Academy of Sciences of the United States of America, 110, 13612-13617. doi:10.1073/pnas.1312477110

Zhao, W., Gong, C., Ouyang, Z., Wang, P., Wang, J., Zhou, P., ... Gong, Z., et al. (2017). Turns with multiple and single head cast mediate Drosophila larval light avoidance. PloS One, 12, e0181193. doi:10.1371/journal.pone.0181193

Zhao, W., Zhou, P., Gong, C., Ouyang, Z., Wang, J., Zheng, N., \& Gong, Z. (2019). A disinhibitory mechanism biases Drosophila innate light preference. Nature Communications, 10, 124. doi:10. 1038/s41467-018-07929-w

Zwart, M.F., Pulver, S.R., Truman, J.W., Fushiki, A., Fetter, R.D., Cardona, A., \& Landgraf, M. (2016). Selective inhibition mediates the sequential recruitment of motor pools. Neuron, 91, 615-628. doi: 10.1016/j.neuron.2016.06.031 